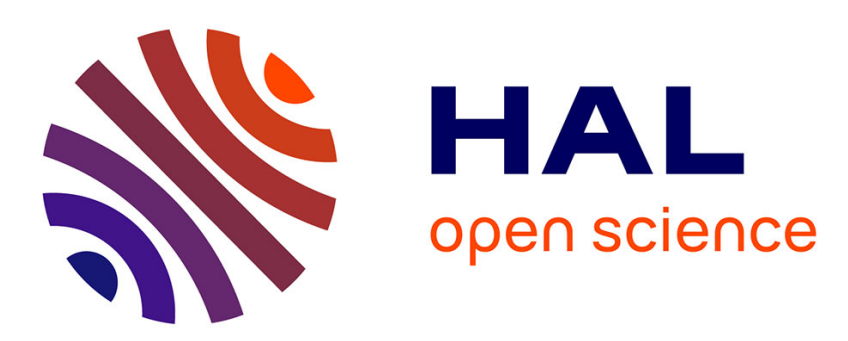

\title{
Differential effect of knee extension isometric training on the different muscles of the quadriceps femoris in humans
}

Giuseppe Rabita, Chantal Pérot, Gishlaine Lensel-Corbeil

\section{- To cite this version:}

Giuseppe Rabita, Chantal Pérot, Gishlaine Lensel-Corbeil. Differential effect of knee extension isometric training on the different muscles of the quadriceps femoris in humans. European Journal of Applied Physiology, 2000, 83, pp.531-538. hal-01561402

\section{HAL Id: hal-01561402 https://hal-insep.archives-ouvertes.fr/hal-01561402}

Submitted on 18 Jul 2017

HAL is a multi-disciplinary open access archive for the deposit and dissemination of scientific research documents, whether they are published or not. The documents may come from teaching and research institutions in France or abroad, or from public or private research centers.
L'archive ouverte pluridisciplinaire HAL, est destinée au dépôt et à la diffusion de documents scientifiques de niveau recherche, publiés ou non, émanant des établissements d'enseignement et de recherche français ou étrangers, des laboratoires publics ou privés. 
Giuseppe Rabita $\cdot$ Chantal Pèrot

Ghislaine Lensel-Corbeil

\section{Differential effect of knee extension isometric training on the different muscles of the quadriceps femoris in humans}

\begin{abstract}
This study determined the effects of a short period of knee isometric training on the quadriceps muscles accessible to surface electromyography (EMG).
\end{abstract}

For this purpose, a training $(n=9)$ and a control $(n=7)$ group were tested on five identical occasions at 1 week intervals during 4 weeks. The training group exercised three times a week by making isometric knee extensions at $80 \%$ of the maximal voluntary contraction (MVC). During the test sessions, maximal and submaximal torque and associated activations of the rectus femoris (RF), vastus lateralis (VL) and vastus medialis (VM) muscles were analysed. As a result of training, differences between MVC values of the two groups were highly significant $(P<0.001)$, whereas only RF-EMG showed significant differences $(P<0.05)$. The VL and VM did not present any significant changes in maximal activation. The EMG-torque relationships were analysed individually before and after the training period. For the control subjects, EMG-torque relationships did not present significant changes while for the training group, these relationships showed a significant increase in RF, VL, and VM maximal activation in 6, 6 and 4 subjects, respectively, and a significant decrease in 1,2 and 5 subjects, respectively. In almost all cases, a significant downward shift of the relationship was observed. This study confirmed that the parts of the quadriceps muscle tested present different adaptation capacities and demonstrate inter-individual variability in the strategies used to enhance muscle strength. In conclusion, to analyse the neural effects resulting from training in a large and compartmentalized muscle like

G. Rabita (C8) - G. Lensel-Corbeil

Laboratoire d'Etudes de la Motricité Humaine,

Faculté des Sciences du Sport et de l'Education

Physique, 59790 Ronchin, France

e-mail: rabita@hp-sc.univ-lille2.fr

Fax: +0033-3-20887377

C. Pè rot

UMR CNRS 6600, Biomé canique et Gé nie Biomé

dical, Universite de Technologie de Compie gne,

B.P. 609, 60206 Compie gne, France the quadriceps femoris, it is desirable to take into account each muscle independently. Moreover, we suggest that overall results obtained from the experiment population should be completed by an analysis on individuals.

Key words Isometric training - Quadriceps muscles • Electromyogram-torque relationships - Surface electromyography

\section{Introduction}

Neural mechanisms have often been considered to be responsible for the increase in muscle strength in the first period of a training programme (Ikai and Fukunaga 1970; Moritani and de Vries 1979; Komi 1986; Sale 1988; Enoka 1997), whereas the muscle factors seem to contribute to training effects only some weeks after the beginning of the period of hyperactivity. Despite the fact that this concept is now generally well recognized, studies on knee extensor muscles trained isometrically have reported an increase in extension torque without notable changes in maximal activation of the quadriceps muscle (Carolan and Cafarelli 1992; Garfinkel and Cafarelli 1992; Weir et al. 1994, 1995). This paradoxical result was partly explained in terms of a decrease in the hamstring muscle co-activation. This result could also be explained by considering that in most of the studies reported above, only the electromyogram (EMG) of the vastus lateralis (VL) or the EMG of both the VL and vastus medialis (VM) were analysed. However, the quadriceps being a large, mixed and compartmentalized muscle, an analysis of its neural adaptation to training needs an evaluation by EMG of the whole activation. However, only three parts of the quadriceps muscle are accessible to surface electrodes. Thus, to test the effects of isometric training, EMG were analysed here from VL, $\mathrm{VM}$ and rectus femoris (RF) muscles as has previously been proposed for dynamic training (Ha' kkinnen and Komi 1983, 1985). This EMG analysis was conducted 
using the methods initially proposed by Moritani and de Vries (1979). These give a representation of the neural and muscle factors of adaptation to training on the basis of EMG-force relationships established before and after the training period. The EMG-torque relationships were obtained from steady (SC) and ramp contractions (RC) using specific software (Pé rot et al. 1996). They concerned each of the three parts of the quadriceps femoris muscle tested and also the sum of the three EMG (Q-EMG). From these different EMG-torque relationships analysed for each subject, it was possible to separate the neural adaptation of the whole quadriceps muscle from each individual part.

\section{Methods}

\section{Subjects}

A group of 16 physical education students volunteered for this investigation and they were divided into a training group $[n=9$, mean age 23.9 (SD 4.8) years, height 169.4 (SD 4.4) $\mathrm{cm}$, body mass $61.4(\mathrm{SD} 7.9) \mathrm{kg}]$ and a control group $[n=7$, mean age 24.0 (SD 5.8) years, height 164.7 (SD 4.3 ) cm, body mass 58.1 (SD 7.0) $\mathrm{kg}]$. The subjects were not involved in any strength training programme.

\section{Training protocol}

The training was conducted three times per week and lasted 4 weeks. The trained subjects performed, bilaterally, isometric strength training of the quadriceps muscles. Each training session began by a warm-up consisting of 5 min of sub-maximal isometric contractions. Following this, five sets of isometric knee extensions were performed at a torque equal to $80 \%$ of the maximal voluntary contraction (MVC) measured at the beginning of each training session. A set consisted of five repetitions with $20 \mathrm{~s}$ of rest between repetitions. Each set was separated by a 2 min rest period. Each repetition lasted $6 \mathrm{~s}$ and was performed at a knee angle of $105^{\circ}$ which allowed the subject to produce his maximal isometric leg extension torque (Thorstensson et al. 1976a).

\section{Test protocol}

The subjects were tested in the same way on five occasions at 1 week intervals during the 4 weeks of the experiment. The first test (T1) was performed before the beginning of the training period while the last test (T5) was performed at the end of the 4 weeks of training. The control group underwent identical tests as the training group but with no additional training. Before T1, all the subjects were carefully familiarized with the test procedure. Each test session began with 2 MVC (4 s SC), then, the subjects performed four RC consisting of $4 \mathrm{~s}$ linear increase in extension torque in the range of $0-100 \%$ MVC. The subjects also performed $4 \mathrm{~s}$ submaximal SC twice at each of $20 \%, 40 \%, 60 \%$ and $80 \%$ MVC. The contractions were requested in a random order.

\section{Torque measurement and display}

During the training and test sessions, the torque was only measured on the right leg but the subject was asked to make symmetrically bilateral contractions to avoid lateral displacements of the pelvis and trunk as had been observed in preliminary unilateral experiments. The pelvis and trunk were firmly secured to the experiment chair of the ergometer in the fashion reported by Gerbeaux et al. (1990). The torque was measured using an ENTRAN strain gauge [type ELF 25
$\mathrm{T}( \pm 250 \mathrm{daN})]$. The subject was positioned so that the ergometer rotation axis was in coincidence with the knee joint rotation axis. An oscilloscope (TEKTRONIX 5113) displayed the torque exerted and the target $\mathrm{RC}$ or SC value to be matched by the subjects.

\section{EMG measurements}

During the test sessions, the EMG activities were recorded from the RF, VL and VM. Bipolar (20 mm inter-electrode distance) surface EMG recording (silver-silver chloride electrodes type Beckmann with $8 \mathrm{~mm}$ active diameter) was employed. For RF, electrodes were placed at mid-distance along the line connecting the anterior-superior-iliac-spine (ASIS) to the superior aspect of the patella. For VL, electrodes were located one quarter of the distance proximal to the lateral tibial condyle on a line connecting this and the ASIS. The electrodes were located over VM at a position approximately $20 \%$ of the distance along a line connecting the medial gap of the knee to the ASIS.

Before application of the electrodes, the skin was thoroughly cleaned by abrasion and sponging with an alcohol-ether mixture to reduce the interelectrode impedance to below $2,000 \mathrm{Q}$.

To ensure the same electrode positions during the 4 weeks of experimentation, marks were made using indelible ink and renewed for each test.

The myo-electrical activities of RF, VL and VM were amplified
VM w passed through upper $(2,000 \mathrm{~Hz})$ and lower $(2 \mathrm{~Hz})$ cut-off filters.

\section{Data analysis}

The EMG and mechanical tracings were stored on a magnetic recorder (BIO-LOGIC DTR 1800) for further analysis using a microcomputer. These signals were digitized with an acquisition card (Direct Memory Access). Each data acquisition was made at a sampling frequency of $1,024 \mathrm{~Hz}$.

During MVC, torque and EMG signals were analysed during the $2 \mathrm{~s}$ window corresponding to the most stable part of the $4 \mathrm{~s} \mathrm{SC}$. During this phase, the EMG activity as root mean square (rms) amplitude was calculated every $500 \mathrm{~ms}$ in consecutive windows. Thus, during a $2 \mathrm{~s}$ window, the mean of the four elementary rms values was taken as the maximal EMG value. The Q-EMG was obtained by summed rms values of the three muscles. In each test session, MVC and EMG values were described as percentages of those obtained in the first test.

The EMG-torque relationships obtained from RC or SC were established using the specific software and methods detailed elsewhere (Ped rot et al. 1996). Briefiy, the mechanical tracings were displayed on the computer monitor. From these mechanical tracings, the experimenter selected the phase of signal to be treated the $4 \mathrm{~s}$ of the RC, then the $2 \mathrm{~s}$ window corresponding to the most stable part of the $4 \mathrm{~s} \mathrm{SC}$. Inside the selected window, the rectified EMG data and the mechanical data were quantified using a sliding average method with a window size of $300 \mathrm{~ms}$ and a window step of $20 \mathrm{~ms}$. Graphics software allowed the display of EMG-torque relationships recorded during $\mathrm{RC}$ or SC. These relationships were displayed using a normalized form: torque and EMG values were related to the highest values $(1,000$ noted) encountered among the signals selected. As previously, the Q-EMG was also analysed from summed rms values of RF, VL and VM-EMG. It was possible to superimpose several relationships on the same graph.

For each subject, EMG-torque relationships obtained at T1 and T5 were compared. The description of the method is presented in Fig. 1.

\section{Statistical analysis}

Two-factor repeated measures analysis of variance (ANOVA) was used to determine differences in MVC at five different times (T1T5). The same analysis was applied to maximal Q-EMG and EMG values of RF, VL and VM. Significant differences were tested using the Scheffe post-hoc test. 




Fig. 1 Evaluation of changes in maximal electromyogram $(E M G)$ values $(a)$ and downward shift $(b)$ of the individual EMG-torque relationships. Pre-post changes in maximal EMG values were evaluated by statistical comparison (Student's $t$-test) between the 15 ordinate points corresponding to the maximal torque observed at test sessions T1 (thin line) and T5 (thick line) (a). Pre-post downward shifts were evaluated by statistical comparison (Student's $t$-test) between the 15 ordinate points corresponding to the same abscissa reference, i.e. the maximal torque obtained at $\mathrm{T} 1(b)$

For individual EMG-torque analysis, differences between values before and after training were tested for significance by Student's $t$-test (paired). For all statistical analyses, the probability level was set at 0.05 .

\section{Results}

Changes in maximal values

Figure 2 shows the changes in the mean MVC values calculated for the trained and the control subjects during the training period. The MVC were significantly higher in the training group after T3 $(P<0.001)$.

For the trained subjects, between T1 and T5, the increase in MCV was $38.7 \%(P<0.001)$. Statistical analysis did not reveal any significant change in the control group.

Figure 3 shows mean maximal values of RF-, VL-, VM- and Q-EMG calculated each week.

For the training group, RF (Fig. 3A) clearly showed a gradual increase in mean activation from week to week. At T5, a significant difference $(P<0.05)$ was observed between RF activity of the trained and the control subjects. While VL activity (Fig. 3B) also showed a gradual increase during the training period, no significant difference was observed between the two groups. Whatever the group tested, EMG values in VM (Fig. 3C) did not show any significant changes. The QEMG analysis (Fig. 3D) did not reveal any significant differences between the trained and the control subjects.

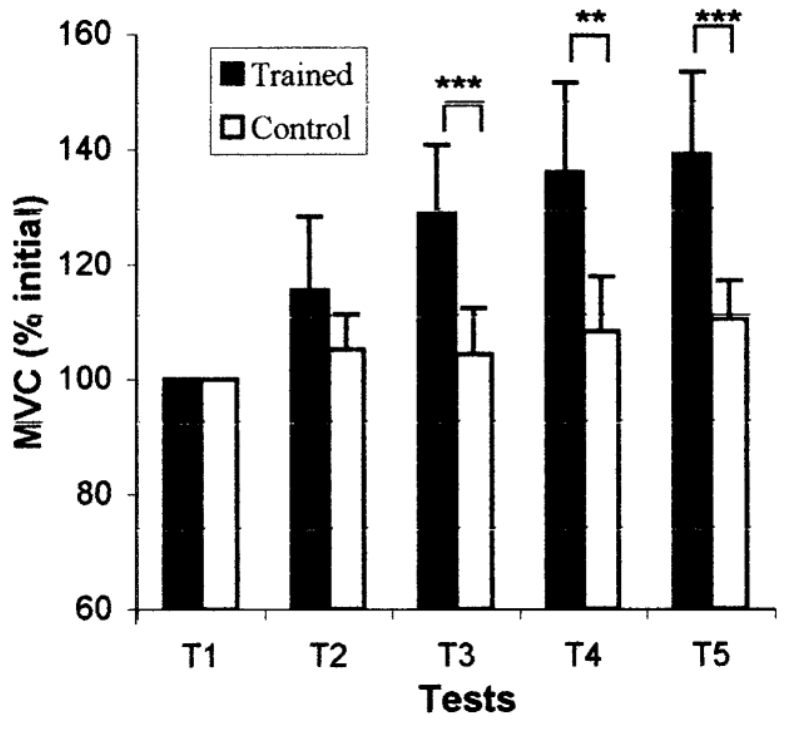

Fig. 2 Isometric maximal voluntary contractions $(M V C)$ during each test (Tl-T5) for the training and the control group. MVC have been expressed as percentages of values measured at the fi test $(T l)$. $* * P<0.01 ; * * * P<0.001$. Differences between the control and the training group

Comparison between EMG-torque relationships obtained in RC and SC

Figure 4 shows, for 1 subject, EMG-torque relationships established for each of the three muscles in RC superimposed with those obtained in SC varying from $20 \%$ to $100 \%$ of MVC. The EMG-torque relationships were curvilinear for each of the three muscles and did not differ for the two types of contractions. Thus, to simplify the presentation of data, only the trials corresponding to RC have been further considered. Figure 4 also shows the scattering of the EMG during SC; this scattering was more pronounced at higher torques. Each subject showed reproducible EMG-torque relationships in RC. The data corresponding to the four RC trials were together fitted to a second order polynomial function. In the following section, only these fitted forms of the EMG-torque relationships were considered in the examination of the effects of training in submaximal and maximal conditions.

Pre-post training changes in EMG-torque relationships

The analysis of the fitted EMG-torque relationships before and after the training period was conducted individually, firstly on each muscle analysed independently (RF-, VL- and VM-EMG) and secondly on the sum of the three muscle activation (Q-EMG).

With regard to the training group, considering the RF muscle, 6 subjects among the 9 showed a significant increase $(P<0.05)$ in maximal EMG values after training and this increase was associated with a significant downward shift of the relationship for 5 of these 

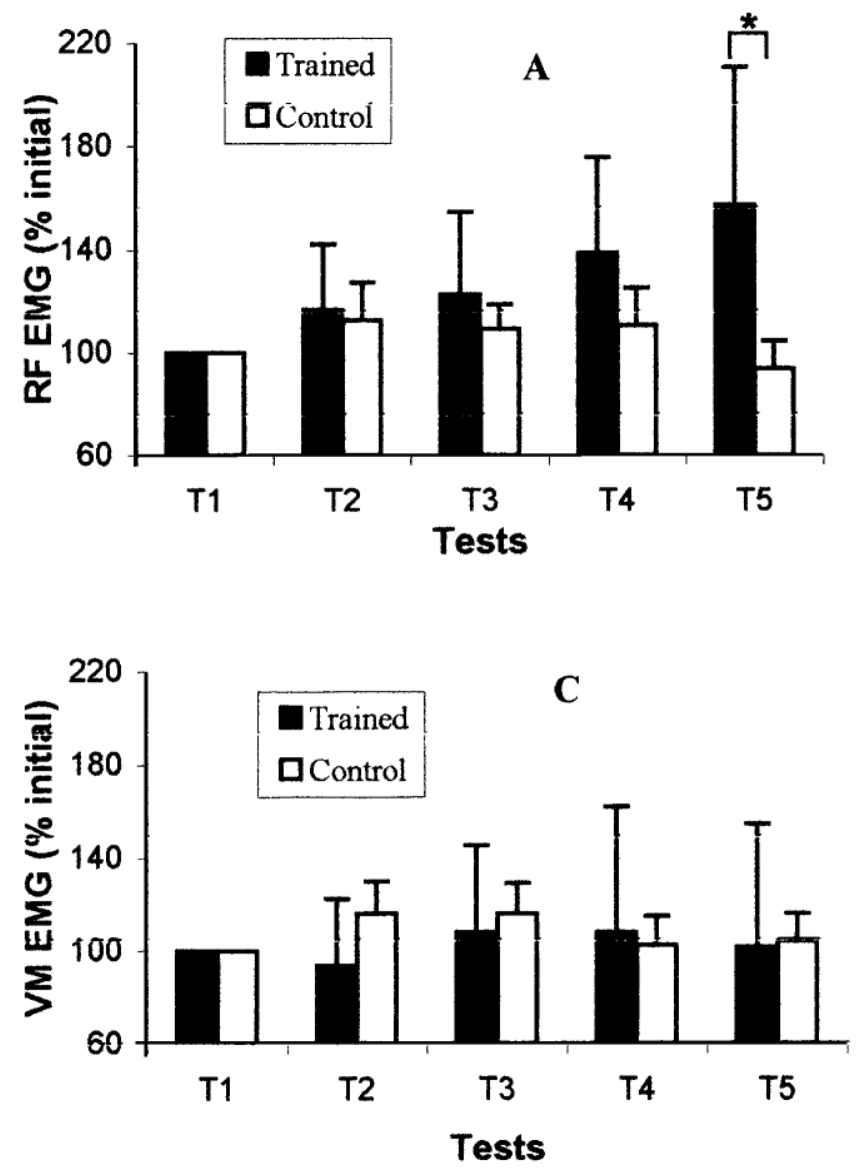

Fig. 3 Maximal electromyogram (EMG) values obtained during each test $(T l-T 5)$ on A the rectus femoris $(R F)$, B the vastus lateralis $(V L)$, $\mathrm{C}$ the vastus medialis $(V M)$ and $\mathrm{D}$ the sum of the three muscle activities $(Q E M G)$. The EMG were expressed as percentages of values measured at the first test $(T l)$. $* P<0.05$ Statistical diff between the control and the training group

subjects (Fig. 5a). Two subjects did not show any significant change in RF maximal activation and 1 subject showed a paradoxical decrease in maximal RF-EMG at T5. These 3 subjects showed a significant downward shift of the relationships.

For the VL muscle, 6 subjects showed a significant increase $(P<0.05)$ in maximal activity after training (Fig. 5b). One subject did not show any significant change between maximal EMG values and 2 subjects showed a decrease $(P<0.05)$ in maximal activation. All the subjects but 2 showed significant downward shift of the relationship.

For the VM muscle, 4 subjects showed a significant increase $(P<0.05)$ in maximal VM-EMG after training but 5 subjects showed the reverse result: a decrease $(P<0.05)$ in maximal VM activation after the training period (Fig. 5c). All the subjects but 1 showed significant downward shift of the relationship.

The Fig. 5d represents a typical result for the control subjects: the changes in both maximal EMG values and downward shift of EMG-torque relationship never reached a significant level, whatever the muscle tested.
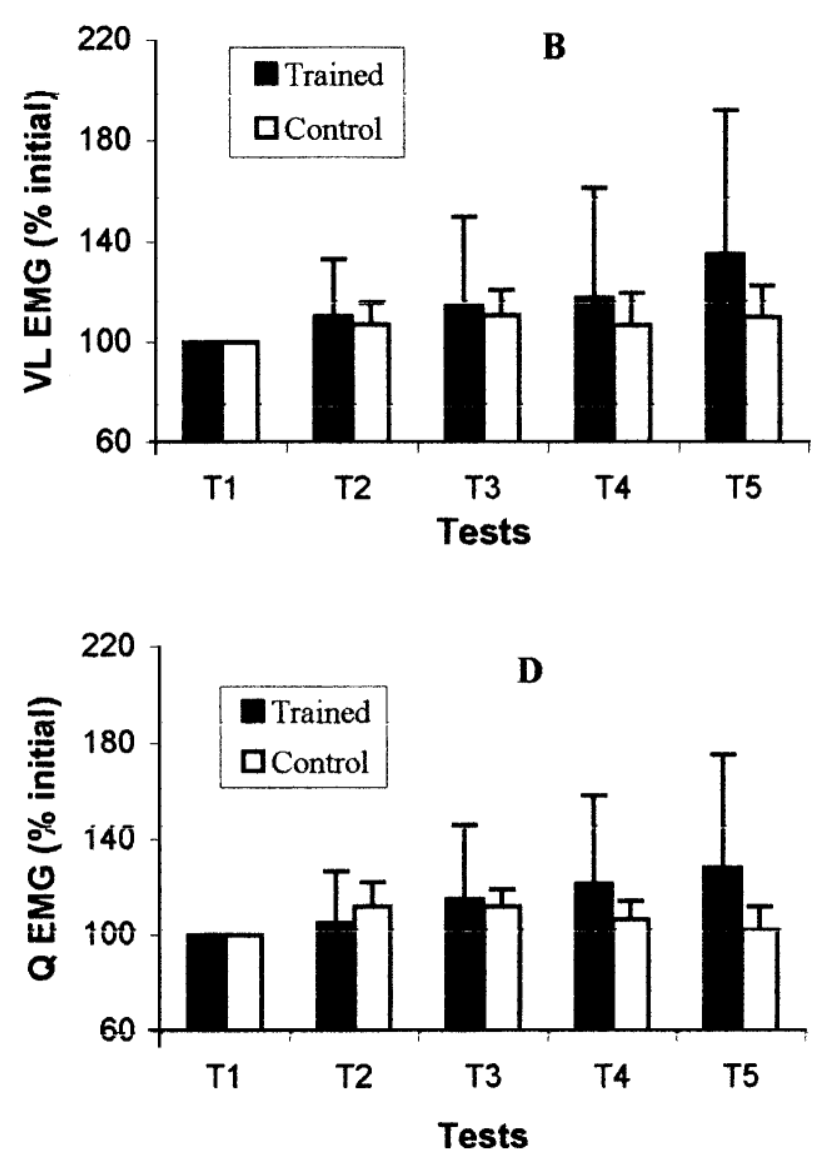

With regard to the Q-EMG-torque relationships obtained in the training group:

1. Only 3 subjects showed an increase $(P<0.05)$ in maximal Q-EMG after training. This increase was associated with a downward shift of Q-EMG-torque relationships for 2 of them (Fig. 6A), whereas for the last, the shift of the relationship was not significant (Fig. 6B).

2. Six subjects did not show any significant change (Fig. 6C) or even show a decrease (Fig. 6D) in maximal Q-EMG between T1 and T5. For all of them, a significant downward shift of the relationship was observed.

For the control subjects, no significant changes were observed in pre-post Q-EMG-torque relationships (Fig. 6E).

\section{Discussion}

The present paper analyses changes in Q-EMG and knee extension torque values throughout a short period of knee extension isometric training, the essential purpose being to differentiate changes in RF, VL and VM activation.

From the second week of training (T3), the analysis of the maximal extension torque revealed a large significant difference between the training and the control 


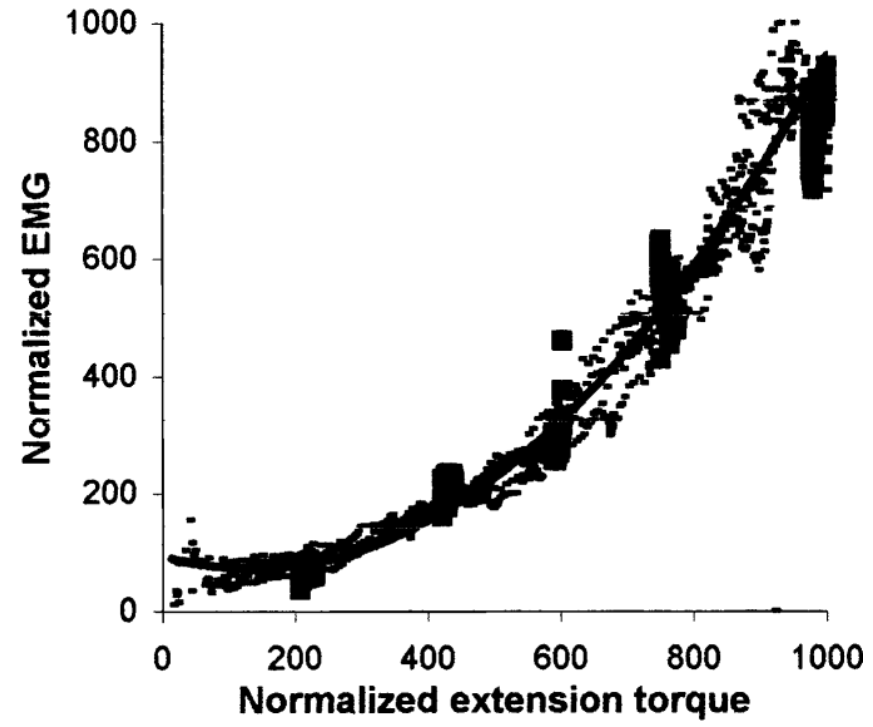

Fig. 4 Example for one subject of electromyogram (EMG)-torque relationships established during four ramp contractions (small squares) and steady contractions (big squares) of the rectus femoris muscle. Torque and $E M G$ were calculated using a sliding average method and related to the highest values (1,000 noted) reached during the experiment. The line drawn represents a 2 nd order polynomial function, fi to the experimental points obtained from the ramp contractions

groups without significant differences in quadriceps activation (Figs. 2, 3). Such results have already been reported by Carolan and Cafarelli (1992) when considering only VL muscle. These authors interpreted these results in terms of a removal of an opposite torque due to a decrease in hamstring co-activation in the early phase of the training period. During the test sessions, each subject was required to perform two unilateral maximal isometric extensions. The MVC and associated EMG obtained during bilateral and unilateral tasks were never found to differ (result not presented). Thus, the bilateral deficit, reported in some cases (Ohtsuki 1983 ; Howard and Enoka 1991) but not in others (Jakobi and Cafarelli 1998), cannot be invoked here to explain the large changes in MVC observed in our study. The analysis of the mean changes in muscle activation during the training period confirmed, for the three quadriceps muscles accessible to surface EMG, previous results obtained when studying only one or two parts of the muscle group. The VM did not present any significant increase in maximal activation confirming the pre-post training study of Weir et al. (1995). For the VL, a non significant increase in activation throughout the training period has been reported by Carolan and Cafarelli 1992 and was also mentioned when considering pre-post effects of a longer ( 8 week) period of isometric training (Garfinkel and Cafarelli 1992; Weir et al. 1994). In contrast, the relatively short period of isometric training was sufficient to induce a gradual increase in RF activation, leading to a significant change as early as 4 weeks after the beginning of the training period. Such
Fig. 5 Examples of fitted electromyogram $(E M G)$-torque relationships established before (T1, thin line) and after (T5, thick line $)$ the training period for three trained subjects $(a, b, c)$ and one control subject (d). The examples of fitted relationships were obtained for the (a) rectus femoris $(R F)(\mathrm{b})$ vastus lateralis $(V L)$ and (c) and (d) vastus medialis $(V M)$. Torque and $E M G$ were related to the highest values (1,000 noted) calculated during either test T1 or $\mathrm{T} 5$
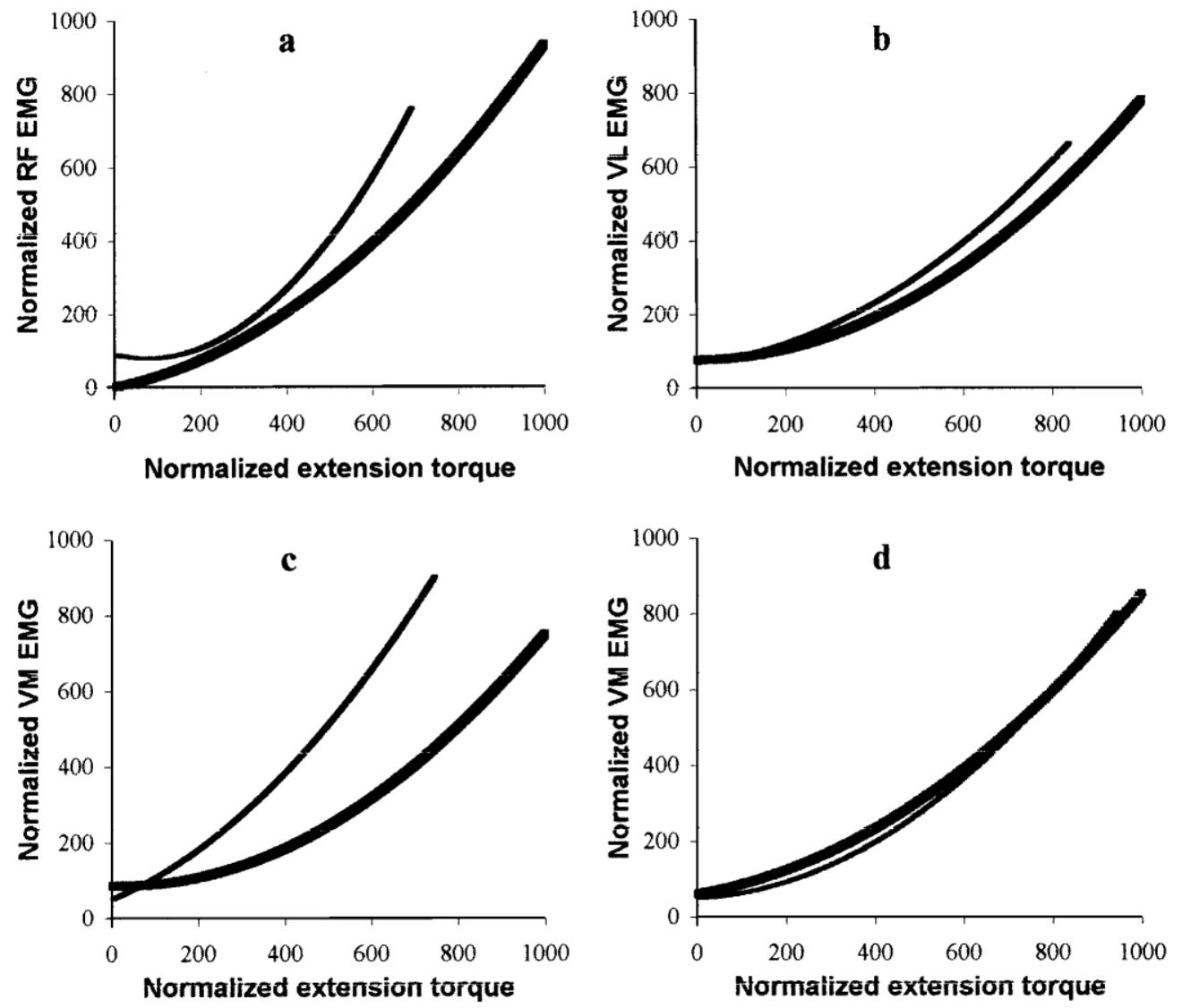
Fig. 6 Example for four trained subjects (A-D) and one control subject (E) of fitted overall quadriceps $(Q)$ EMGtorque relationships established before (T1, small squares) and after (T5, big squares) the training period. Torque and QEMG were related to the highest values $(1,000$ noted $)$ calculated during either test, $\mathrm{T} 1$ or T5
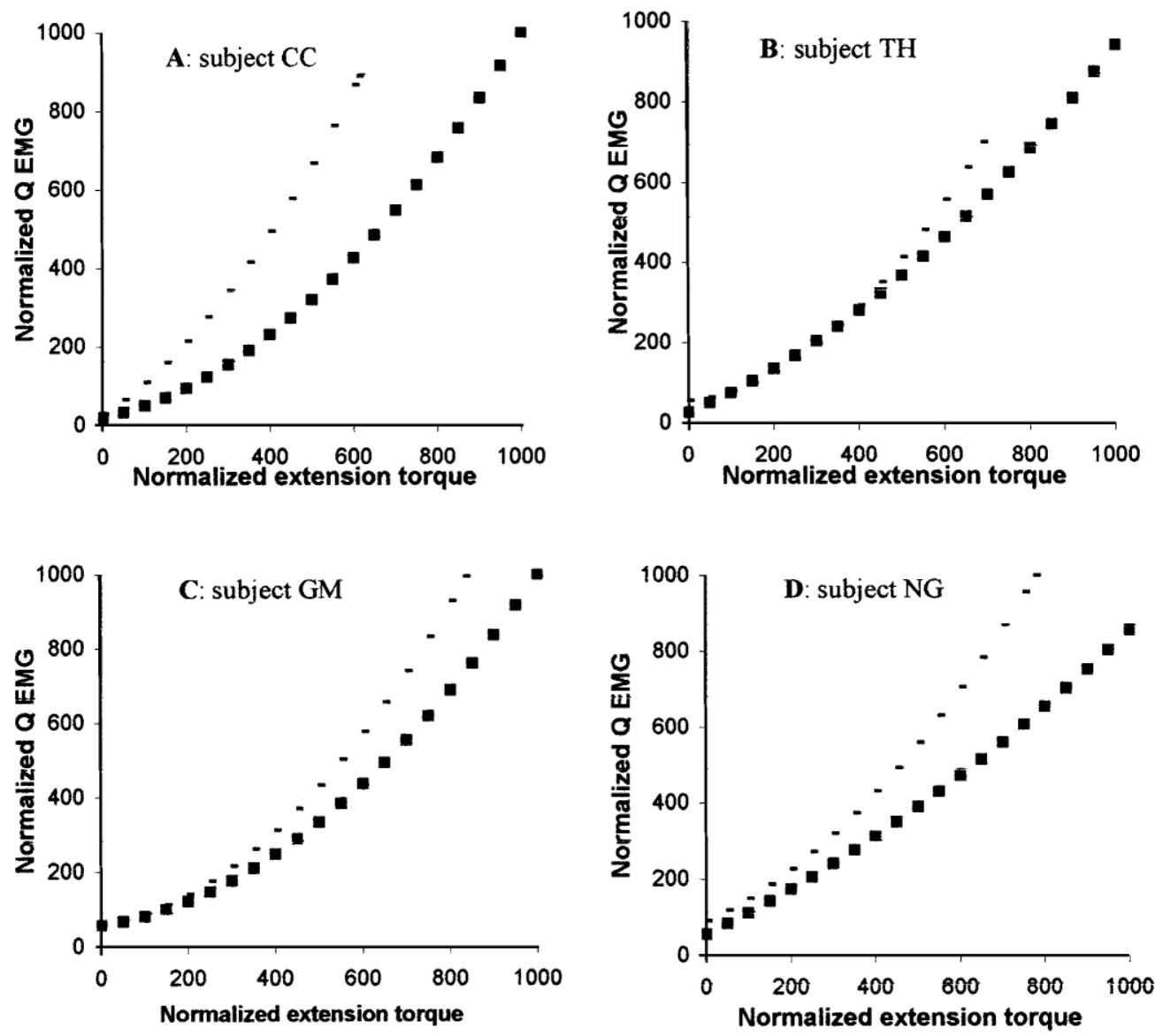

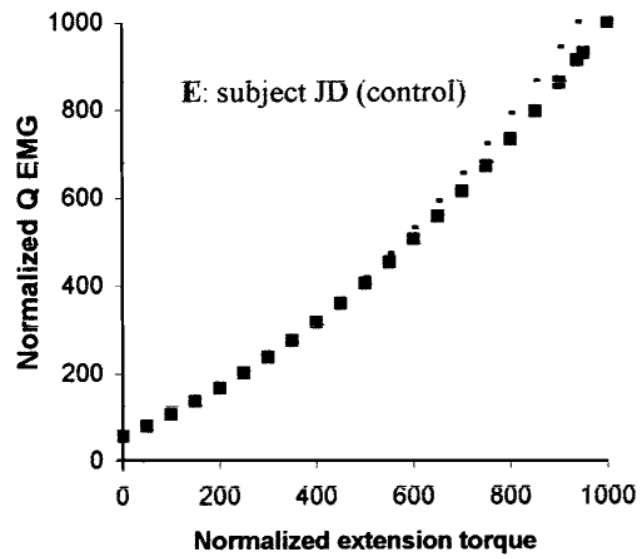

an increase in RF-EMG with isometric training was previously reported by Komi et al. (1978).

Thus, the present study confirmed that the RF muscle presents the higher adaptation capacities after a short period of isometric training whereas mean values obtained for the trained population did not reveal significant changes in VL and VM maximal levels of activation. This higher adaptation of RF compared to the other quadriceps muscles could also refiect the fact that, in the sitting position, RF, a bi-articular muscle, was trained at a shorter relative length than the monoarticular VL and VM. Indeed, these anatomical considerations could be related to the fact that as training and test sessions were performed at the same joint position an angular specificity could be present. The - paut-Mathieu et al. (1988) provided some EMG evi- dence indicating a greater increase in motor unit activation at the joint angles trained. Furthermore, this angular specificity has been reputed to be more marked at shorter muscle lengths (The paut-Mathieu et al. 1988; Kitai and Sale 1989). These findings are in favour of a greater activation of the muscle trained in the shorter position, i.e. the RF muscle.

The effect of the isometric training was more precisely differentiated by the analysis, for each subject and each muscle, of EMG-torque relationships estab- 
lished before and after training. The model proposed by Moritani and de Vries (1979) allows the differentiation of neural and muscle factors contributing to the torque gain in maximal and submaximal contractions. For that purpose, normalized EMG-torque relationships established before and just after training were superimposed on the same graph. On the basis of linear EMG-torque relationships, Moritani and de Vries (1979) attributed the increase in torque resulting from training to:

1. Purely neural factors when an increase in maximal EMG values was observed after training without change in the slope of the relationship

2. Purely muscle factors when a decrease in slope was observed without changes in maximal EMG values

3. Both neural and muscle factors when both increase in maximal EMG values and decrease in slope were observed.

The shape of the EMG-torque relationships obtained in our study being almost always curvilinear, muscle factors were not appreciated by studying the decrease in slope but the downward shift of the relationship. Furthermore, the effects of training on the EMG-torque relationships were analysed from data recorded during RC after checking (Fig. 4) that the RC or SC did not have any infiuence either on the maximal values or on the shape of the relationships. These identical shapes of the EMG-torque relationships in $\mathrm{RC}$ or $\mathrm{SC}$ have already been reported for the biceps brachii muscle (Pè rot et al. 1996).

With regard to the EMG-torque relationships, no control subject showed significant differences between the first and the last test. This attested the reproducibility of the EMG measurements and ruled out the possible infiuence of the repetitive testing on the results.

According to the methodology of Moritani and de Vries (1979), a strengthening could be attributed to both neural and muscle adaptations if both an increase in maximal Q-EMG and a downward shift of the relationships were observed. This result was observed here for only 2 subjects of the training group, while the majority of these trained subjects showed a downward shift of Q-EMG-torque relationships without enhancement in maximal Q-activation. Similar results were obtained by Ha' kkinen and Komi (1983) and Ha' kkinen et al. (1985) after dynamic training. In those studies, the long duration of training (16 and 24 weeks, respectively) al- lowed muscle hypertrophy to develop and the explana- tion of the adaptation only in terms of muscle factors. In contrast, the short training period of our study prevents us from attributing the downward shift of the relation- ships to muscle hypertrophy, as muscle factors have been shown to begin to contribute to the effects of training only some weeks after the beginning of hyper- activity (Moritani and de Vries 1979; Komi 1986; Sale 1988; Enoka 1997). In the same way as mentioned above, to explain changes in maximal torque values, the downward shift of the relationships may be better explained in terms of changes in muscle coordination and synergies. The non-increase in maximal Q-activation observed for the majority of the subjects did not signify that the different parts of the quadriceps were not affected by the training period. The individual EMGtorque relationships often revealed a decrease in neural activation in one or two muscles (mainly VM or VL) whereas another component showed an increase after training. This paradoxical decrease in neural activation was previously reported as a result of a dynamic training study (Thorstensson et al. 1976b) and could explain why Q-EMG, the sum of the $3 \mathrm{EMG}$, did not change for some subjects.

This study suggests that the training effects vary notably from one part of the muscle to another and from one subject to another. This high variability could be explained by the fact that the quadriceps is a large muscle having several components. Thus, the neural control of such a muscle is necessarily more complex than for a small, non-compartmentalized muscle containing a relatively small number of motor units. This would explain the fact that when considering mean values calculated for the population, we failed to observe any change in quadriceps muscle activation. These unchanging mean values could in fact refiect opposite activation changes in different parts of the muscle of different individuals. Our results illustrate the fact that, when considering only the first few weeks of a training period, it is necessary to complete the analysis of changes obtained for the population by considering also the analysis of data from individuals. Indeed, neural adaptation is susceptible to presenting inter-individual variability. This variability in neural strategies being also valid when considering the different components of the quadriceps muscle, the EMG analysis of training effects should not be limited to any one component, unrepresentative of the whole muscle.

In conclusion, the present knee isometric training study resulted in an increase in knee extension torque unaccompanied by changes in neural activation of the whole quadriceps muscle. These observations seem partly to account for a differential effect of training in the individual components of the quadriceps muscle: only RF muscle showed a significant increase in activation after the 4 week training period. The results obtained for the whole quadriceps muscle as well as for the individual components (RF, VL and VM) confirmed the compartmentalization of training effects and showed inter-individual variability in the strategies used to enhance muscle strength. It is proposed that, to analyse the neural effects resulting from training in a large and compartmentalized muscle like the quadriceps femoris, it is desirable to take into account each component muscle independently. Moreover, we suggest that overall results for experiment populations should be presented only after an analysis has been conducted on individuals. 


\section{References}

Carolan B, Cafarelli E (1992) Adaptation in coactivation after isometric resistance training. J Appl Physiol 7: 911-917

Enoka RM (1997) Neural adaptation with chronic physical activity. J Biomech 30: 447-455

Garfinkel S, Cafarelli E (1992) Relative changes in maximal force, EMG, and muscle cross-sectional area after isometric training. Med Sci Sport Exerc 24: 1220-1227

Gerbeaux M, Lensel-Corbeil G, Pertuzon E (1990) Exploration des mouvements: principes de conception d'un ergome tre. In: Nougier V, Blanchi JP (eds) Pratiques sportives et mode lisation du geste. Collection Grenoble Sciences, Grenoble, pp 377-394

Ha' kkinen K, Komi PV (1983) Electromyographic changes during strength training and detraining. Med Sci Sport Exerc 15: $455-460$

Ha' kkinen K, Komi PV (1985) Changes in electrical and mechanical behavior of leg extensor muscles during heavy resistance strength training. Scand J Sports Sci 7: 5564

Hä' kkinen K, Alen M, Komi PV (1985) Changes in isometric force- and relaxation-time, electromyographic and muscle fibre char- acteristics of human skeletal muscle during strength training and detraining. Acta Physiol Scand 125: 573585

Howard JD, Enoka RM (1991) Maximum bilateral contractions are modified by neurally mediated interlimb effects. J Appl Physiol 70: 306-316

Ikai M, Fukunaga T (1970) A study on training effect on strength per unit cross-sectional area of muscle by mean of ultrasonic measurement. Int Z Angew Physiol 28: 173-180

Jakobi JM, Cafarelli E (1998) Neuromuscular drive and force production are not altered during bilateral contractions. J Appl Physiol 84: 200-206

Kitai TA, Sale DG (1989) Specificity of joint angle in isometric training. Eur J Appl Physiol 58: 744-748
Komi PV (1986) Training of muscle strength and power: interaction of neuromotoric, hypertrophic, and mechanical factors. Int J Sports Med 7: 10-15

Komi PV, Viitasalo JT, Rauramaa R, Vihko V (1978) Effect of isometric strength training on mechanical, electrical, and metabolic aspects of muscle function. Eur J Appl Physiol 40: 45-55

Moritani T, De Vries HA (1979) Neural factors versus hypertrophy in the time course of muscle strength gain. Am J Phys Med 58: $115-130$

Ohtsuki T (1983) Decrease in human voluntary isometric arm strength induced by simultaneous bilateral exertion. Behav Brain Res 7: 165-178

Pé rot C, Andre L, Dupont L, Vanhoutte C (1996) Relative con- tributions of the long and short heads of the biceps brachii during single or dual isometric tasks. J Electromyogr Kinesiol 6: 3-11

Sale DG (1988) Neural adaptation to resistance training. Med Sci Sport Exerc 20: S135-S145

The paut-Mathieu C, Van Hoecke J, Maton B (1988) Myoelectrical and mechanical changes linked to length specificity during isometric training. J Appl Physiol 64: 15001505

Thorstensson A, Grimby G, Karlsson J (1976a) Force-velocity relations and fibre composition in human knee extensor muscles. J Appl Physiol 40: 12-16

Thorstensson A, Karlsson J, Viitasalo JT, Luhtanen P, Komi PV (1976b) Effect of strength training on EMG of human skeletal muscle. Acta Physiol Scand 98: 232-236

Weir JP, House TJ, Weir LL (1994) Electromyographic evaluation of joint angle specificity and cross-training after isometric training. J Appl Physiol 77: 197-201

Weir JP, House TJ, Weir LL, Johnson GO (1995) Effects of unilateral isometric strength training on joint angle specificity and cross-training. Eur J Appl Physiol 70: 337-343 